\title{
The Ileal Gastrointestinal Stromal Tumor: A Rare Site of Occurrence
}

\author{
Ahsan Zil-E-Ali ${ }^{*}$, Ali Akram Khan ${ }^{2}$, Syeda Naqvi ${ }^{3}$ \\ ${ }^{1}$ Departments of Surgery, Fatima Memorial Hospital, Lahore, Pakistan \\ ${ }^{2}$ Internal Medicine, Detroit Medical Center, Wayne State University, Michigan, USA \\ ${ }^{3}$ Medicine, Jinnah Sindh Medical University, Karachi, Pakistan
}

"Corresponding author: Ahsan Zil E Ali, Department of Surgery, Fatima Memorial Hospital, Lahore, 725 Shadman Rd, Lahore 54000, Pakistan. Tel: +9242111555600; Email: ahsanzileali@gmail.com

Citation: Ali AZE, Khan AA, Naqvi S(2017) The Ileal Gastrointestinal Stromal Tumor: A Rare Site of Occurrence. J Surg: JSUR-160. DOI: $10.29011 /$ JSUR-160.000060

Received Date: 05 August 2017; Accepted Date: 28 August 2017; Published Date: 04 September, 2017

\begin{abstract}
Gastrointestinal Stromal (GIST) tumors are one of the common malignancies arising from mesenchymal tissue. They can be present anywhere in the gastrointestinal tract, most commonly in the stomach and small intestine; less frequent in colon, rectum, and esophagus. We describe a rare presentation of GIST tumor in Ileum in a young patient without any risk factor and positive family history. Furthermore, this patient responded well to surgical resection and Imatinib therapy. Due to high recurrence rate, five years follow up with annual whole body Computed Tomography scan (CT) was advised.
\end{abstract}

\section{Introduction}

GIST tumor was the term first coined by Mazur and Clark in 1983.They originate from Cajal cells, which are pacemakers for gut peristalsis, present in myenteric plexus of the gastrointestinal tract. In 1998, mutation of KIT was recognized as one of the causes of GIST tumors. In 2001, selective inhibitors of KIT and tyrosine kinase like Imatinib were introduced [1]. Most GIST tumors are sporadic in occurrence, rarely also associated with syndromes like Neurofibromatosis, Familial GIST, Carney's Triad and CarneyStratakis syndrome. The common types of GIST are spindle, epithelioid and mixed. The immunohistochemical stain like KIT (CD117) is a sensitive marker for the diagnosis [2]. Mostly GIST tumors, if symptomatic presents with gastrointestinal bleeding as the most common symptom. Tumors less than $6 \mathrm{~cm}$ tends to remain asymptomatic or an incidental finding. If symptomatic management options are surgical resection of the tumor. In addition to that preoperative Imatinib can also be given to enhancing tumor shrinkage before surgery. After surgery, the recurrence rate is around $70 \%$ in 5 years and one can predict a higher chance of recurrence by high mitotic activity (can be predicted by Ki-67)[1,2].

\section{Case Report}

A 38 years old man presented with right hypochondriac pain with nausea for past 6 months and multiple vomiting episodes during this time, along with dark stool on some occasions and early morning abdominal tenderness. The patient had fatigue and malaise along with the gastrointestinal discomfort. Patient's family history was positive for colorectal and breast cancer. He is a smoker, one pack per day for last five years.On physical examination, he had pale conjunctivae and signs of anemia. On deep palpation of the abdomen, no major gross abnormality was appreciated. His baselines including Complete Blood Count, Liver function test and Gastrointestinal series were ordered. Those were significant for anemia and a pelvic mass. A computerized tomography was ordered to confirm the diagnosis and it showed a huge mass. A surgical consult was offered and surgical removal of the mass was proposed as the management option. The patient consented to the procedure and general anesthesia. A routine pre-operative protocol for blood workup was carried, which was normal. 'Battle' incision was planned and the lower GI tract was explored for the mass. The mass was arising from ileal loops abutting the sigmoid colon in its plane and compressing the dome of the bladder. The mass was highly angiogenic, with many vessels visible on gross presentation. A careful surgical resection from the ileal wall was done. The mass was sent for the histopathological analysis that showed a gastrointestinal stromal tumor on hematoxylin and eosin stain with c-kit/CD117 positive on immunohistochemistry. An oncologist was involved and imatinib was given as a pharmacological treatment to prevent recurrence. The occurrence at the aforementioned age and the site located, makes it a peculiar and different than the classical presentation. It is also notable that the mass revealed on CT image could have led to other gross abnormalities, which were absent. 


\section{Discussion}

Gastrointestinal Stromal Tumor (GIST) is the commonest mesenchymal tumor of the gastrointestinal tract. These are submucosal lesions frequently growing endophytically but can also manifest exophytically. These tumors are believed to originate from the intestinal pacemaker cell, i.e. the intestinal cells of Cajal. The most common sites are stomach (40-60\%) and small intestine (30-40\%), while less frequent sites include the colon and rectum.

Sizes of these tumors vary from as small as $1 \mathrm{~cm}$ in diameter to as large as $40 \mathrm{~cm}$. The worldwide incidence of this tumor is 11-19.6 per a million people, GISTs represent just $1 \%$ of all gastrointestinal primary tumors. Typically, GIST affects people over 50 years of age, rarely those under age 40 , and the average age of diagnosis is around 63 years old $[3,4]$. This is peculiar to find a tumor at a younger age as the case presented by us. The symptoms and presentations of GISTs are variable and depend on the location, size, the relationship with the gastrointestinal wall, and malignancy. When these tumors grow to a certain size, GI bleeding may result from ulceration of the mucosal surface or abdominal pain may arise from the compression of surrounding tissues or organs. The most common symptoms or presentations associated with GISTs are vague, nonspecific abdominal pain or discomfort. Patients may describe a feeling of fullness and rarely, or an abdominal mass may be palpable.

GISTs may also produce symptoms secondary to obstruction or hemorrhage. Patients who have experienced significant blood loss may report malaise, fatigue, or exertional dyspnea. At the same time, patients may present with obstructive symptoms such as dysphagia, constipation, and obstructive jaundice, depending on whether the tumor is present in esophagus, colon or duodenum respectively [2]. The gain of functional mutation in the KIT (c-kit)/CD117 proto-oncogene represents about 95\% of GIST. The Interstitial Cells of Cajal (ICC) are the pacemaker cells of the bowel and are c-kit/CD117 positive, as was the case with our patient. 2 Commonly expressed but less GISTs-specific antigens are CD34, nestin, Smooth Muscle Actin (SMA), caldesmon, calponin, vimentin, and embryonic smooth muscle myosin. Off these CD34 is found in $80-85 \%$ of Gastric GISTs and about $50 \%$ of intestinal GISTs [3]. Diagnosis can also be established through upper GI endoscopy; alternately CT and MRI have been used as modalities to diagnose GISTs. Even though CT and MRI have been the best imaging modality for assessment of primary lesion and detection of metastases, they are usually not helpful in specifying the origin of the mass. Management is primarily surgical. Surgical resection should include en-bloc removal with the pseudo capsule intact. Imatinib is approved by the FDA for use in unresectable and/or metastatic KIT-positive GIST in the United States and Europe. Preoperative management with Imatinib in patients with inoperable or malignant GIST may enable these patients to undergo subsequent curative resection $[2,3]$. Sunitinib has been used as a second line therapy for metastatic disease as well as in cases with Imatinib resistance.

The risk of recurrence is determined by the size of the tumor. Tumors that are less than $2 \mathrm{~cm}$ have a very low risk of recurrence where as those more than $10 \mathrm{~cm}$ have a very high recurrence risk. GISTs are usually very soft and friable and rupture easily, but tumor rupture during surgery is correlated with an extremely high risk of recurrence.

Up to two-thirds of patients with GISTs will have metastasis or recurrence. The median time for recurrence is 19 to 25 months; however, follow up for recurrence is long term as metastasis have been reported to occur as late as 30 years after the removal of the primary tumor. ${ }^{2}$ The overall relative 5-year survival rate of people diagnosed with a malignant GIST was estimated to be about $76 \%$. In the case where the tumor was confined to the organ where it started, the 5-year relative survival was $91 \%$. When the same tumor spread into nearby tissue (and/or lymph nodes) the survival rate went down to $74 \%$ while with distant sites the 5-year survival rate was $48 \%[5,6]$.

The best and definitive plan is a surgical intervention. From year 2000 to 2005, under the objective of assessing the viability and success rate of laproscopic technologies application and surgical excision of gastrointestinal stromal tumors, 10 out of a total of 28 patients who were operated upon for GIST, underwent laproscopic gastric resection with tumor surgery. The analysis demonstrated that following such a procedure, the recurrence of disease in the next 2-5 years follow-up was not present, which in turn, establishes that laparoscopic operations have an added benefit over open interventions while keeping the oncological radicalism intact [7]. Surgery remained the only option of treatment till 2001. It was followed by a five-year survival rate of around $50 \%$. However, the recent development of use of TKI drugs for example Imatinib has improved patient prognosis while simultaneously increasing the probability of R0 resection. Although, GIST tumors are still likely to recur in a small percentage of patients, known as the high-risk group. And that is why the emphasis on follow-up during this postsurgical period cannot be iterated upon enough. Some relevant studies are available but there are no risk stratification criteria that has been able to establish gastrointestinal bleeding caused by GIST as a significant indicator. Careful assessment of data regarding prognosis of Gastrointestinal hemorrhage caused by GIST led to the conclusion that since patients with gastrointestinal bleeding caused by hemorrhage are more likely to suffer from recurrent tumors, careful follow-up in them is absolutely essential. Therefore, patients with gastrointestinal bleeding are considered a high-risk group, and doctors treating such conditions should be well-aware of the possibility and risk of post-operative recurrence.

The relevant Kaplan-Meier curves show that the 1) 
recurrence-free survival and 2) the overall survival, both are shorter for patients with gastrointestinal bleeding caused by GIST. Gastrointestinal bleeding is an independent risk factor for GIST recurrence and death of the patients and should be considered a significant indicator of poor prognosis [8]. it is wise to discuss the possibility and viability of robot-assissted surgeries which have gained enormous acceptance among surgeons. The reason of their popularity is that they provide highly magnified three-dimensional image view that improves hand-eye coordination. Such systems provide beneficial effects including those in the range of motion scaling, multi-dimensional stereoscopic visualization, excellent instrument dexterity with tremor fibrillation which ensures accuracy of precise movements in tight spaces. This advent not only solves many problems regarding surgical expertise but also overcomes human error while providing better precision. However, a study performed in Italy brought forth some limitations of the procedure where robot-assisted surgeries did not readily manage cases of minimal bleeding. Although there were no fatalities, it did contribute to the morbidity. It has been established that to shift the learning curve to master, 10 solo surgeries must be performed alongside a fellow robot. The factors limiting the success rate of this expensive procedure include limited arms on the robot, lesser popular academic training as well as large diameters of the instrument $[9,10]$.

It is recommended that a thorough evaluation is needed with right diagnostic tests and more importantly the appropriate intervention. Keeping in mind the prognostication, one must keep imatinib and other similar chemotherapies in point of discussion while explaining the disease to the patient (Figure 1-4).

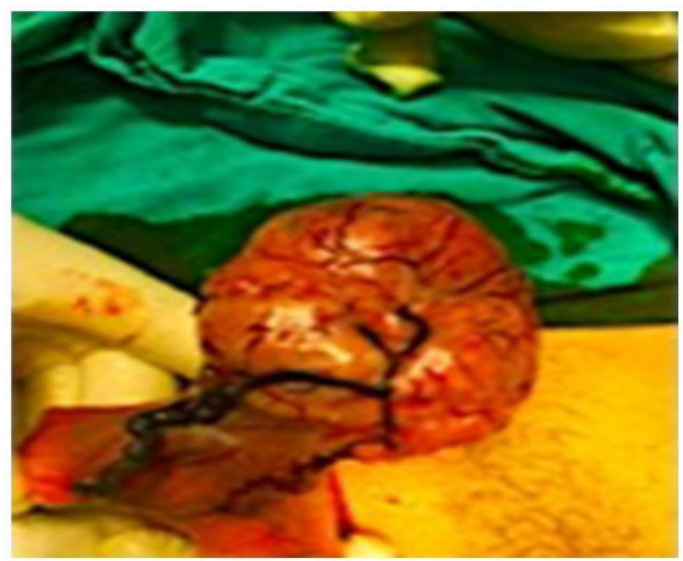

Figure 1: Intra-operative visualization of GIST tumor from the ileum with high vasculature visible.

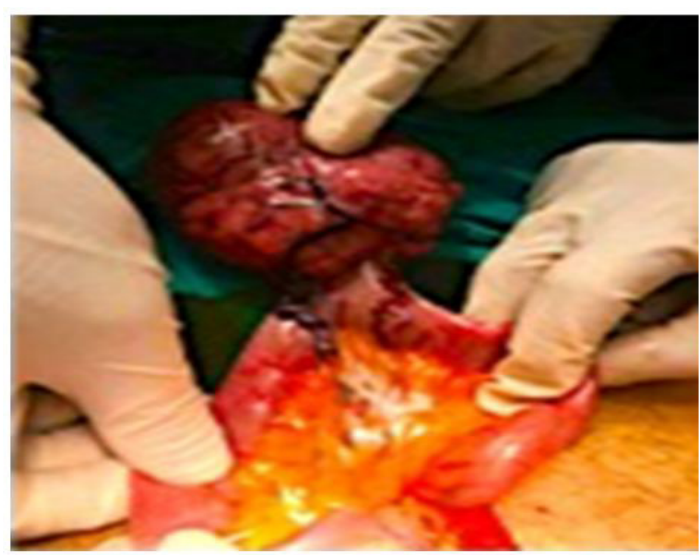

Figure 2: Intra-operative handling of the tumor within the ileal loops.

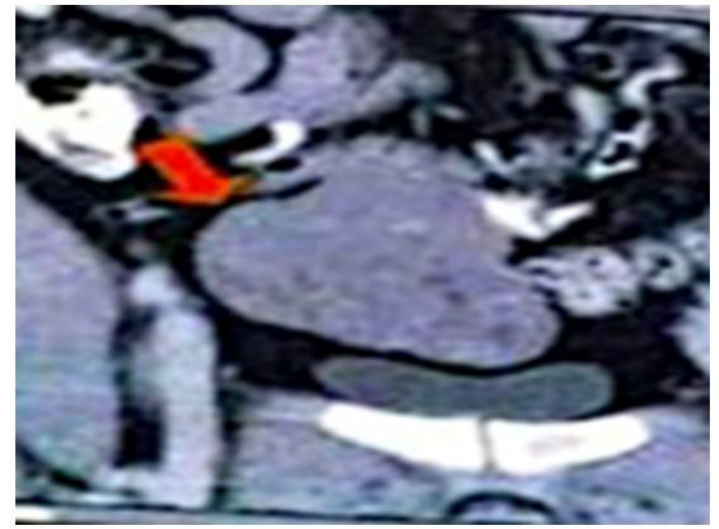

Figure 3: CT scan image of the ileal GIST tumor compressing the adjacent tissue and protruding into pelvic area.

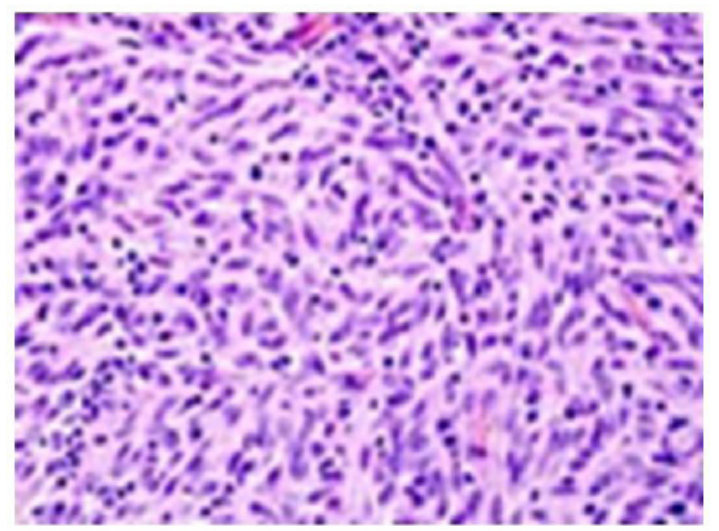

Figure 4: Histopathological picture showing whorled pattern and high $\mathrm{N}: \mathrm{C}$ ratio cells. 
Citation: Ali AZE, Khan AA, Naqvi S(2017) The Ileal Gastrointestinal Stromal Tumor: A Rare Site of Occurrence. J Surg: JSUR-160.

\section{References}

1. Laperouse P, Raines D, Diamond K, Rivera S, Newman W, et al. (2008) Journal of the Louisiana State Medical Society 160.

2. Joensuu H, Hohenberger P, Corless CL (2013) Gastrointestinal stromal tumour. Lancet 382:973-983.

3. López A, Vargas R, Mejía J, Pérez L, García ARevistaMédica del Hospital General de México.

4. Sashidharan P, Matele A, Matele U, Al Felahi N, Kassem K(2014) Gastrointestinal Stromal Tumors: A Case Report Oman Medical Journal 29: 138-141.

5. Tan C, Zhi W, Shahzad G, Mustacchia P (2012) Gastrointestinal Stromal Tumors: A Review of Case Reports, Diagnosis, Treatment, and Future Directions. International Scholary Research Notices 2012.

6. Xiang Yu Q, Kun He Z, Wang J, Sun C, Zhao W, et al. (2014) Clinical presentations of gastric small gastrointestinal stromal tumors mimics functional dyspepsia symptoms.World J Gastroenterol 20: 1180011807.
7. Grubnik VV, Kovahichuk AL, Malinovskiy AV, Barannikov KV (2016) Possibilities of laparoscopic gastric resection for gastrointestinal stromal tumors. KlinKhir 2016: 5-7.

8. Liu Q, Li Y, Dong M, Kong F, Dong Q (2017) Gastrointestinal Bleeding Is an Independent Risk Factor for Poor Prognosis in GIST Patients. BioMed Research International 2017.

9. Song SH, Lee C, Jung J, Kim SJ, Park S, et al. (2017) A comparative study of pediatricopen pyeloplasty, laparoscopy-assisted extracorporeal pyeloplasty, and robot-assisted laparoscopic pyeloplasty. PLoS One 12.

10. Zil-E-Ali A, Assad S, Jabbar F (2017) Simultaneous Robot-assisted Laparoscopic Excision of Pre-pyloric Gastrointestinal Stromal Tumor and Cholecystectomy. Muacevic A, Adler JR, eds. Cureus 9:e1306. 